\title{
Epidemiological features and spatio-temporal clusters of hand-foot-mouth disease at town level in Fuyang, Anhui Province, China (2008-2013)
}

\author{
Y. J. MAO ${ }^{1}$, L. SUN ${ }^{2}$, J. G. XIE ${ }^{1 *}$ AND K. K. W. YAU ${ }^{3}$ \\ ${ }^{1}$ School of Management, University of Science and Technology of China, Hefei, Anhui Province, China \\ ${ }^{2}$ Fuyang Centre for Diseases Control and Prevention, Fuyang, Anhui Province, China \\ ${ }^{3}$ Department of Management Sciences, City University of Hong Kong, Hong Kong, China
}

Received 3 August 2015; Final revision 13 July 2016; Accepted 13 July 2016;

first published online 1 August 2016

\section{SUMMARY}

Hand-foot-mouth disease (HFMD) is a frequently occurring epidemic and has been an important cause of childhood mortality in China. Given the disease's significant impact nationwide, the epidemiological characteristics and spatio-temporal clusters in Fuyang from 2008 to 2013 were analysed in this study. The disease exhibits strong seasonality with a rising incidence. Of the reported HFMD cases, $63 \cdot 7 \%$ were male and $95 \cdot 2 \%$ were preschool children living at home. The onset of HFMD is age-dependent and exhibits a 12-month periodicity, with 12-, 24- and 36-month-old children being the most frequently affected groups. Across the first 60 months of life, children born in April [relative risk (RR) 8·18], May (RR 9.79) and June (RR 8.21) exhibited an elevated infection risk of HFMD relative to January-born children; the relative risk compared with the reference (January-born) group was highest for children aged 24 months born in May (RR 34.85). Of laboratory-confirmed cases, enterovirus 71 (EV71), coxsackie A16 (Cox A16) and other enteroviruses accounted for $60 \cdot 1 \%, 7 \cdot 1 \%$ and $32 \cdot 8 \%$, respectively. Spatiotemporal analysis identified one most likely cluster and several secondary clusters each year. The centre of the most likely cluster was found in different regions in Fuyang. Implications of our findings for current and future public health interventions are discussed.

Key words: Age (months), birth month, epidemiological features, hand-foot-mouth disease, onset month, spatio-temporal cluster.

\section{INTRODUCTION}

Hand-foot-mouth disease (HFMD) is caused by a group of enteroviruses, with the most common ones being enterovirus 71 (EV71) and coxsackie A16 (Cox A16) [1]. EV71 is more strongly associated with severe cases compared to Cox A16 [1]. The latent period of HFMD is 2-7 days, and patients normally

\footnotetext{
* Author for correspondence: Dr. J. G. Xie, Associate Professor, School of Management, University of Science and Technology of China (USTC), 96 Jin Zhai Road, Bao He District, China. (Email: xiej@ustc.edu.cn)
}

recover in 7-10 days [2]. Most affected individuals can recover without complications. However, many severely ill patients develop fulminant cardiorespiratory failures, which are often fatal [1]. The majority of affected cases are aged $<60$ months, but adults can also be infected. Precautionary measures, such as avoiding close contact with infected individuals and maintaining good hygiene, are effective means of preventing the spread of the disease [2].

HFMD epidemics have escalated in the Asia-Pacific region since the 1990s, with EV71-associated HFMD occurring in regular cycles [3]. A total of 29 patients died between April and June in Malaysia in 1997, 
with the disease primarily caused by EV71 [4]. In 1998, a large EV71 epidemic occurred in Taiwan, where 405 severe complications were recorded, of whom 78 died [5]. The largest Asia-Pacific pandemic was reported in China in 2008, posing a challenge to public health. The disease first appeared in Fuyang, Anhui Province, then rapidly developed into a nationwide epidemic, with around 490000 cases and 126 deaths reported [3]. HFMD was categorized as a class $\mathrm{C}$ infectious disease by the Ministry of Health of China on 2 May 2008. Since then, medical institutions have been required to report HFMD cases within $24 \mathrm{~h}$.

In 2009, the incidence rate of HFMD in mainland China was about 8.66/10 000 (1 155525 cases), and its fatality rate was $0 \cdot 03 \%$ (353 deaths) [1]. Better understanding of the transmission dynamics and epidemiological characteristics of HFMD may provide useful guidance in implementing preventive measures to control its outbreaks and minimize morbidity and mortality. The present study describes HFMD distribution with respect to age (year and month), birth month, time, area, etc., over the period 2008-2013. We aimed to assess the spatio-temporal distribution of HFMD.

\section{MATERIALS AND METHODS}

\section{Study area}

Fuyang is located northwest of Anhui Province, Eastern China $\left(114^{\circ} 52^{\prime} \mathrm{E}\right.$ to $116^{\circ} 49^{\prime} \mathrm{E}, 32^{\circ} 25^{\prime} \mathrm{N}$ to $34^{\circ} 004^{\prime} \mathrm{N}$ ). It is situated near the southern fringe of the warm temperate zone; its annual average temperature is $\sim 15.0^{\circ} \mathrm{C}$, and the annual average precipitation is $907 \mathrm{~mm}$. Fuyang has the largest population among the cities in Anhui Province according to the sixth National Census in 2010.

Fuyang has 172 subdistrict offices, villages, and towns managed by three municipal districts (Yingzhou, Yingquan, Yingdong), four counties (Yingshang, Taihe, Linquan, Funan), and one county-level city (Jieshou). In this study, the three municipal districts were regarded as urban, and the other parts of Fuyang were regarded as rural according to administrative jurisdiction and economic development level. In China, subdistrict offices, villages, and towns are of the same administrative rank and are referred to in the present paper as 'town level'.

\section{Data collection}

HFMD surveillance data from 2008 to 2013 were provided by Fuyang Centre for Diseases Control and
Prevention (CDC). Ethical approval and patients' consent were obtained via the CDC's standard protocol for data collection. The data included patients' gender, birth date, onset date, diagnosis date, severity level, current address, etc. The geographical coordinates of the study area were obtained from Google Earth, and the administrative map of Fuyang at the town level was supplied by Fuyang Civil Affairs Bureau. Patient onset date was selected as the HFMD occurrence date; addresses and coordinate information were linked with the Fuyang administrative map. Demographic data at the county level were acquired from the 2014 Anhui Statistical Yearbook; however, accurate population data at the town level were unavailable. As a proxy, we divided a county's population by the number of towns and this value was taken as an estimate of the mean population of each town within the county, then HFMD incidence rate at town level was calculated.

\section{Descriptive epidemiology analysis}

The incidence rate of HFMD was calculated annually and by area. The analyses of demographic characteristics were conducted by year. The number of reported cases was summarized monthly and by age to describe the temporal and age distribution of HFMD. The number of months between birth month and onset month was calculated as the patient's age in months. Patients aged $<60$ months were classified into 144 groups by their birth month and onset month (12 birth-month groups, 12 onset-month groups). Then onset-month distribution was described for each birthmonth group. The majority of cases were children aged $<60$ months who accounted for $96 \cdot 1 \%$ (102 405/106 565) of the total number of cases over the entire study period; hence, age 60 months was selected as the upper age threshold in analyses regarding patients' age in months, birth month, onset month and isolated virus serotype.

To assess the impact of birth month and age on infection risk, patients born in 2008 were selected as the analysis sample and the number of births in 2008 was summarized monthly. We assumed that there was no HFMD-related population migration over the study period. The surveillance data recorded the comprehensive information about all reported HFMD cases from 2008 to 2013 in Fuyang. Therefore, for individuals who were born in 2008, the standard information was recorded in our surveillance data if they were infected and hospitalized with HFMD in their 
first 60 months of life. The absolute risk of infection was calculated for each birth-month group in 2008 (e.g. the number of HFMD patients born in January divided by the number of births in January). We then quantified the relative risks (RRs) of HFMD infection for each birth-month group and peak age groups with $95 \%$ confidence intervals (CIs). January was selected as the reference month for each age and birth-month group because of the lowest incidence.

\section{Spatio-temporal cluster analysis}

The space-time scan statistic was introduced by Kulldorff et al. in 1998; Kulldorff's SaTScan (http:// www.satscan.org/) is one of the most popular software packages for the space-time scan statistic method [6]. The space-time statistic is defined by a cylindrical window, with the base referring to the circular geographical region and the height representing time. The window undergoes dynamic changes in its base and height to detect potential spatio-temporal clusters [7]. SaTScan scans for clusters of geographical size between zero and a pre-set maximum value. The maximum cluster size can be specified as a percent of population at risk in the study, or in terms of geographical size using the circle radius. The cluster radius is the circular size of a detected cluster, a geographical region is included in the cluster if its centroid is included in the scanning circle [8].

The space-time scan statistic can be used for prospective and retrospective analyses based on several types of probabilistic models, such as discrete/continuous Poisson model, discrete Bernoulli model, and spacetime permutation model. At present, the discrete Poisson and space-time permutation models are extensively adopted in cluster detection and early warning of different kinds of diseases, such as cervical cancer, brain cancer, and HFMD [6, 7, 9]. The main difference between these two models lies in their initial data requirements. The former requires the demographic data of study areas, whereas the latter does not; therefore, the latter is especially practical when population information is inaccessible. Hence, the space-time permutation model was utilized in this study to detect spatio-temporal areas in Fuyang with abnormally high HFMD incidence.

SaTScan detects possible clusters by calculating the $\log$ likelihood ratio (LLR) for each cluster as follows:

$$
\operatorname{LLR}=\log (c / n)^{c}[(C-c) /(C-n)]^{C-c},
$$

where $C$ is the total number of cases, $c$ is the observed number of cases within the scan window, and $n$ is the expected number of cases within the window. The relative risk (RR; equivalent to $c / n$ ) and the $P$ value of the LLR are calculated for each cluster. The scan window with maximum significant LLR is considered the most likely cluster; other scan windows with significant LLR are identified as secondary clusters and ranked according to their LLR test statistic $[7,8,10]$. In this study, clusters with $P$ values $<0.001$ were reported.

SaTScan has two limitations. First, the software lacks cartographic support. Thus, scan results cannot be visualized by this software. Second, scan results are very sensitive to parameter settings [6]. To overcome these limitations, ArcGIS 10.2 (ESRI, USA) was utilized to visualize scan results, and a method for optimal selection of parameters was employed in this study as follows.

No consensus exists regarding the selection of parameters in SaTScan; most previous studies set the maximum spatial cluster size (scan radius) to $50 \%$ and/or $20 \%$ of the total population at risk; the maximum temporal cluster size was set to $50 \%$ of the study period $[9,11-13]$. However, scan results tend to include large cluster areas and regions where no significant elevated risk exists by using $50 \%$ as the threshold value [14]. Scan results are closely related to parameter settings. The hotspots and scope of infected areas may keep changing over the entire study period. Thus, the optimal scan radii should probably be changed every year instead of being kept constant. An exploratory analysis was performed by using the retrospective space scan statistic based on the discrete Poisson model to determine favourable parameters [15]. Specifically, the retrospective space scan statistic based on the discrete Poisson model was employed to analyse 2008 county-level HFMD data in Fuyang. The maximum circular size of the scanning window was set to the default value, which is $50 \%$ of the total population at risk. The results of this method showed that the maximum cluster size was $29.82 \mathrm{~km}$; that is, the largest cluster would be detected if the scan radius was set to $29.82 \mathrm{~km}$. Therefore, when an optimal setting for the retrospective space-time scan permutation statistic was determined, 30, 25, and 20 $\mathrm{km}$ were selected as the upper bounds of the scan radius. The corresponding maximum temporal cluster size was set to 7 days (the incubation period of HFMD is $2-7$ days, and according to the Guidelines for Prevention and Control of Hand, Foot and Mouth Disease formulated by the Ministry of Health of China in 2009, the cluster of HFMD cases is defined within 7 days). 


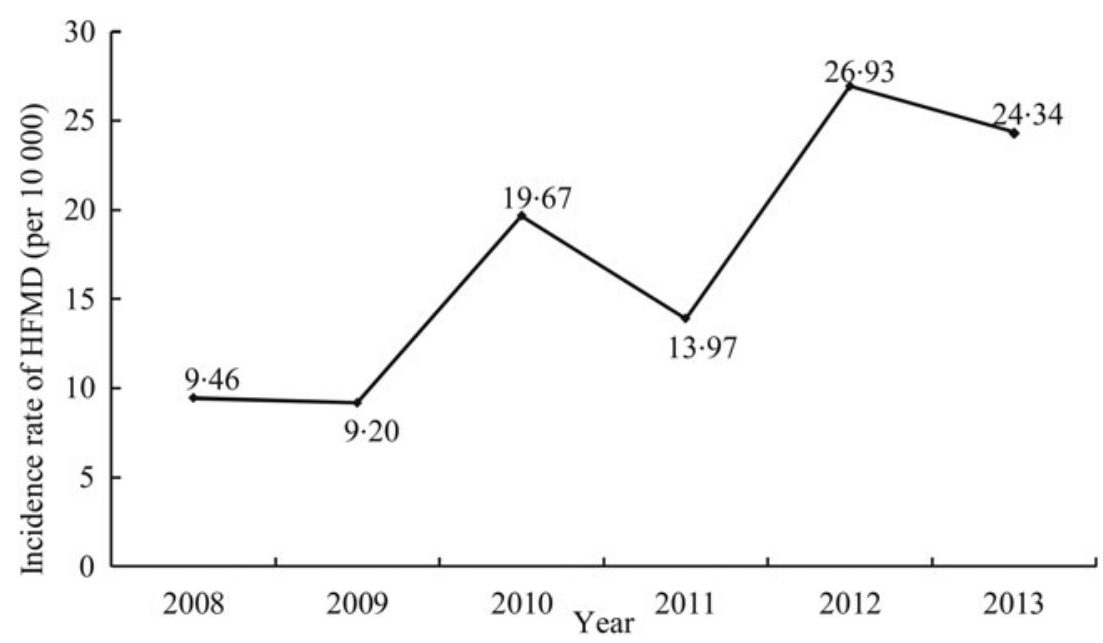

Fig. 1. Incidence rate of hand-foot-mouth disease (HFMD) in Fuyang from 2008 to 2013 (per 10000 ).

Tango \& Takahashi [16] recommend that the number of regions included in the largest cluster should be deemed unfavourable when it exceeds $10-15 \%$ of the total number of regions. The total number of geographical regions in the present study is 172 . Therefore, the number of regions included in the largest cluster should be $<25$. The results obtained with different scan radii show that the scan radius of $20 \mathrm{~km}$ satisfied the required criteria (the number of regions included in the largest cluster was 15). Similarly, this approach was employed to obtain favourable parameter choices for the remaining 5 years of the study. The optimal scan radii were set as $30 \mathrm{~km}$ in $2009,15 \mathrm{~km}$ in $2010,20 \mathrm{~km}$ in 2011 , and $15 \mathrm{~km}$ in 2012 and 2013. The maximum temporal cluster size was set to 7 days for all study years.

\section{RESULTS}

\section{Incidence of HFMD}

As shown in Figure 1, the incidence rate of HFMD demonstrated a rising trend over the 6-year study period (the $\chi^{2}$ for trend statistic is 12460 with $P<0 \cdot 001$ ), increasing from $9 \cdot 46 / 10000$ in 2008 to $24 \cdot 34 / 10000$ in 2013. More specifically, in 2009 the morbidity of HFMD was only marginally smaller than that in 2008. After that, the rate increased markedly to $19 \cdot 67$ in 2010, which was twofold higher than that in the preceding year. After declining to 13.97 in 2011, the next year recorded a sharp rise again, reaching the highest point at 26.93 and then finally the incidence rate dropped slightly in 2013 to $24 \cdot 34$. Overall, during the study period, HFMD generally grew and reached a peak every other year.

\section{Temporal distribution}

As shown in Figure 2, the monthly distribution of HFMD exhibited strong seasonality, with seasonal peaks occurring between April and June in almost every year since 2008 .

\section{Demographic characteristics}

Table 1 summarizes the demographic characteristics of the HFMD cases. A total of 106565 HFMD cases were reported in Fuyang from 2008 to 2013, including $67927(63 \cdot 7 \%)$ male patients and 38638 $(36 \cdot 2 \%)$ female patients. The male-to-female ratio ranged from 1.6:1 to 1.9:1 (the average ratio was 1.8:1, cf. Table 1). According to the Anhui Statistical Yearbook, the proportion of male population was slightly higher than that of female over the study period $(52.0 \%$ and $48.0 \%$, respectively). Children aged $<60$ months predominated in reported cases. In terms of patient status, Table 1 shows that most of the cases were preschool children who lived at home or attended kindergarten $(95 \cdot 2 \%$ and $3 \cdot 5 \%$, respectively). The proportion of students and others are relatively small (students are defined as those who receive formal education in school or other places. The rest of patients are categorized as other cases). In total, the number of HFMD cases in rural areas was higher than that of urban areas.

\section{Birth month and onset month distribution}

The relationship between patient's birth month and month of illness onset is shown in Figure 3. Each cell in Figure $3 a$ represents the number of patients who were 


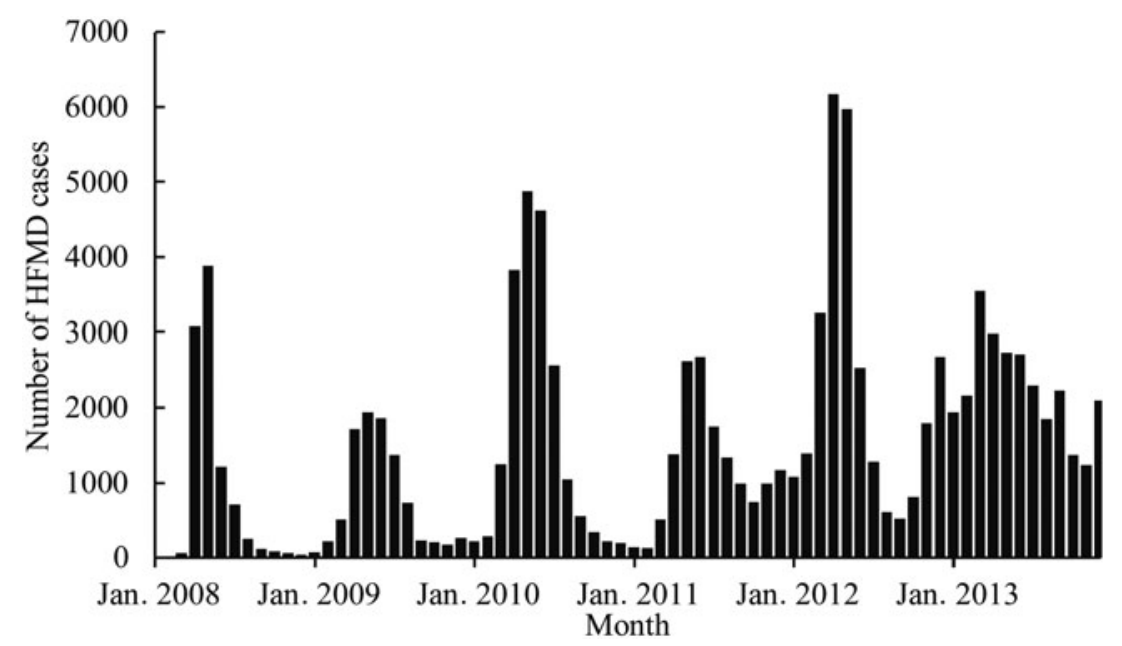

Fig. 2. Temporal distribution of hand-foot-mouth disease (HFMD) in Fuyang from 2008 to 2013.

born in $m$ and developed HFMD in $n$, i.e. the size of previously mentioned 144 groups ( $n, m=$ January, February, ..., December). As shown in Figure $3 a$, a large number of patients were born between April and June. Each row in Figure $3 b$ shows the onset-month distribution for each birth-month group (the sum of the percentages in each row is $100 \%$ ). For instance, the first cell in Figure $3 b$ represents patients born in January who developed HFMD in January and accounts for 53.9\% of the total number of January-born patients (2683/ 4979; 4979 is the sum of numbers in the first row in Fig. $3 a$ ). It was found from Figure $3 b$ that more than half of the patients were identified as having HFMD in the month they were born (the diagonal from upper left to lower right in Fig. $3 b$, the proportion ranges from $52 \cdot 5 \%$ to $89 \cdot 0 \%$ ). In addition, an increased chance of infection occurred during the peak months of HFMD, i.e. April-June (percentages in the fourth to sixth column in Fig. $3 b)$ as well as in the month before the birth month. For example, in the February birth group (the second row in Fig. 3b), 62.2\% of patients developed HFMD in February, $5 \cdot 9 \%$ in April, $7 \cdot 2 \%$ in May, $4 \cdot 3 \%$ in June, and $4.0 \%$ in January.

\section{Age distribution}

The age distribution of HFMD patients was investigated and the results are presented in Figure 4. The age (months) distribution plot reveals a particular trend with strong periodicity every 12 months in individuals with HFMD in Fuyang. Three large peaks occurred when the patients were aged 12, 24 and 36 months. Medium peaks appeared at the 48th and 60th month after birth, with several small peaks occurring before age 11 months and at 11 th the $23 \mathrm{rd}$ and 35th month of life.

\section{Impact of birth month and age}

Figures 3 and 4 indicate that birth month and age might be important risk factors of HFMD. The absolute risk and relative risk of HFMD infection for each birth-month group are presented in Table 2. Birth month is associated with increased HFMD risk in the first 60 months of life, with April, May and June being the peak birth-month risk groups. In particular, $34.5 \%$ of children born in May 2008 were diagnosed with HFMD in their first 60 months of life, compared to $3.5 \%$ of children born in January 2008 (RR 9.79, 95\% CI 8.88-10.79).

As shown in Figure 5, in peak age groups (children aged 12, 24, 36, 48, 60 months), 12-, 24-, and 36-month-olds born between April and June had a statistically significant elevated risk of HFMD infection compared to children of the same ages born in January. In fact, for those aged 12, 24, 36, and 48 months, most birth-month groups had significantly higher risk than in January. The largest increment in risk of HFMD infection compared to the reference January birth group was age 24 months, born in May (RR 34.85, 95\% CI 26.49-43.25), while age 60 months, born in August (RR 0.21, 95\% CI 0.08-0.55) exhibited the lowest risk.

\section{Virus serotype distribution}

In practice, given the limited laboratory capacity, most HFMD cases are retrieved from clinical reports, 
Table 1. Demographic characteristics of reported HFMD cases in Fuyang from 2008 to 2013

\begin{tabular}{|c|c|c|c|c|c|c|c|}
\hline & \multicolumn{7}{|l|}{ Year, $N(\%)$} \\
\hline & $\begin{array}{l}2008 \\
(N=9441)\end{array}$ & $\begin{array}{l}2009 \\
(N=9216)\end{array}$ & $\begin{array}{l}2010 \\
(N=19908)\end{array}$ & $\begin{array}{l}2011 \\
(N=14349)\end{array}$ & $\begin{array}{l}2012 \\
(N=28017)\end{array}$ & $\begin{array}{l}2013 \\
(N=25634)\end{array}$ & $\begin{array}{l}\text { Total } \\
(N=106565)\end{array}$ \\
\hline \multicolumn{8}{|l|}{ Gender } \\
\hline Male & $6124(64 \cdot 9)$ & $6085(66 \cdot 0)$ & $12850(64 \cdot 5)$ & $9368(65 \cdot 3)$ & $17723(63 \cdot 3)$ & $15777(61 \cdot 5)$ & $67927(63 \cdot 7)$ \\
\hline Female & $3317(35 \cdot 1)$ & $3131(34 \cdot 0)$ & $7058(35 \cdot 5)$ & $4981(34 \cdot 7)$ & $10294(36 \cdot 7)$ & $9857(38 \cdot 5)$ & $38638(36 \cdot 3)$ \\
\hline Male/female ratio & $1 \cdot 8: 1$ & $1 \cdot 9: 1$ & $1 \cdot 8: 1$ & $1 \cdot 9: 1$ & $1 \cdot 7: 1$ & $1 \cdot 6: 1$ & $1 \cdot 8: 1$ \\
\hline \multicolumn{8}{|l|}{ Age group } \\
\hline $0-60$ months & $8818(93 \cdot 4)$ & $8836(95.9)$ & $19033(95 \cdot 6)$ & $13857(96 \cdot 6)$ & $26862(95 \cdot 9)$ & $24999(97 \cdot 5)$ & $102405(96 \cdot 1)$ \\
\hline$>60$ months & $623(6 \cdot 6)$ & $380(4 \cdot 1)$ & $875(4 \cdot 4)$ & $492(3 \cdot 4)$ & $1155(4 \cdot 1)$ & $635(2 \cdot 5)$ & $4160(3 \cdot 9)$ \\
\hline \multicolumn{8}{|l|}{ Status } \\
\hline Preschool children living at home & $7475(79 \cdot 2)$ & $8555(92 \cdot 8)$ & $18608(93 \cdot 5)$ & $13960(97 \cdot 3)$ & $27480(98 \cdot 1)$ & $25402(99 \cdot 1)$ & $101480(95 \cdot 2)$ \\
\hline Children attending kindergarten & $1625(17 \cdot 2)$ & $461(5 \cdot 0)$ & $1002(5 \cdot 0)$ & $219(1 \cdot 5)$ & $310(1 \cdot 1)$ & $120(0 \cdot 5)$ & $3737(3 \cdot 5)$ \\
\hline Students & $312(3 \cdot 3)$ & $180(2 \cdot 0)$ & $268(1 \cdot 3)$ & $131(0 \cdot 9)$ & $185(0 \cdot 7)$ & $84(0 \cdot 3)$ & $1160(1 \cdot 1)$ \\
\hline Others & $29(0 \cdot 3)$ & $20(0 \cdot 2)$ & $30(0 \cdot 2)$ & $39(0 \cdot 3)$ & $42(0 \cdot 1)$ & $28(0 \cdot 1)$ & $188(0 \cdot 2)$ \\
\hline \multicolumn{8}{|l|}{ Virus serotype } \\
\hline Coxsackie A16 & $1(0 \cdot 0)$ & $15(0 \cdot 2)$ & $7(0 \cdot 0)$ & $12(0 \cdot 1)$ & $32(0 \cdot 1)$ & $6(0 \cdot 0)$ & $73(0 \cdot 1)$ \\
\hline Enterovirus 71 & $26(0 \cdot 3)$ & $50(0 \cdot 5)$ & $78(0 \cdot 4)$ & $218(1 \cdot 5)$ & $114(0 \cdot 4)$ & $134(0 \cdot 5)$ & $620(0 \cdot 6)$ \\
\hline Other enterovirus & $0(0 \cdot 0)$ & $12(0 \cdot 1)$ & $20(0 \cdot 1)$ & $58(0 \cdot 4)$ & $129(0 \cdot 5)$ & $120(0 \cdot 5)$ & $339(0 \cdot 3)$ \\
\hline Missing data & $9414(99 \cdot 7)$ & $9139(99 \cdot 2)$ & $19803(99 \cdot 5)$ & $14061(98 \cdot 0)$ & $27742(99 \cdot 0)$ & $25374(99 \cdot 0)$ & $105533(99 \cdot 0)$ \\
\hline \multicolumn{8}{|l|}{ Region } \\
\hline Urban area & $4699(49 \cdot 8)$ & $2852(30 \cdot 9)$ & $7902(39 \cdot 7)$ & $4815(33 \cdot 6)$ & $9073(32 \cdot 4)$ & $9996(36 \cdot 9)$ & $39337(36 \cdot 9)$ \\
\hline Rural area & $4742(50 \cdot 2)$ & $6364(69 \cdot 1)$ & $12006(60 \cdot 3)$ & $9534(66 \cdot 4)$ & $18944(67 \cdot 6)$ & $15638(60 \cdot 1)$ & $67228(63 \cdot 1)$ \\
\hline
\end{tabular}


(a)

Month of illness onset

\begin{tabular}{|c|c|c|c|c|c|c|c|c|c|c|c|c|c|}
\hline & Jan. & Feb. & Mar. & Apr. & May & June & July & Aug. & Sep. & Oct. & Nov. & Dec. & Number \\
\hline Jan. & 2683 & 32 & 120 & 377 & 427 & 271 & 247 & 158 & 133 & 106 & 129 & 296 & \\
\hline Feb. & 204 & 3143 & 121 & 297 & 363 & 217 & 161 & 109 & 103 & 93 & 90 & 148 & \\
\hline Mar. & 97 & 352 & 6548 & 327 & 310 & 239 & 163 & 105 & 115 & 60 & 105 & 120 & \\
\hline Apr. & 66 & 59 & 659 & 13506 & 260 & 170 & 107 & 57 & 67 & 60 & 64 & 105 & \\
\hline May & 54 & 64 & 227 & 1528 & 15482 & 216 & 127 & 70 & 70 & 44 & 55 & 80 & \\
\hline June & 36 & 60 & 177 & 358 & 1058 & 11021 & 90 & 72 & 53 & 46 & 55 & 106 & \\
\hline July & 32 & 48 & 166 & 302 & 452 & 829 & 6951 & 49 & 32 & 25 & 29 & 53 & \\
\hline Aug. & 23 & 49 & 147 & 332 & 446 & 373 & 508 & 3969 & 48 & 33 & 35 & 61 & \\
\hline Sep. & 20 & 34 & 149 & 302 & 455 & 329 & 252 & 334 & 3162 & 21 & 31 & 61 & \\
\hline Oct. & 14 & 16 & 140 & 384 & 496 & 356 & 287 & 203 & 300 & 2492 & 21 & 41 & \\
\hline Nov. & 10 & 8 & 99 & 253 & 496 & 314 & 280 & 216 & 192 & 257 & 3295 & 24 & \\
\hline Dec. & 8 & 31 & 79 & 210 & 388 & 317 & 204 & 176 & 162 & 152 & 359 & 5085 & \\
\hline
\end{tabular}

(b)

Month of illness onset

\begin{tabular}{|c|c|c|c|c|c|c|c|c|c|c|c|c|c|}
\hline & Jan. & Feb. & Mar. & Apr. & May & June & July & Aug. & Sep. & Oct. & Nov. & Dec. & \multirow{12}{*}{$\begin{array}{l}\text { Proportion } \\
\square\end{array}$} \\
\hline Jan. & $53.9 \%$ & $0.6 \%$ & $2 \cdot 4 \%$ & $7 \cdot 6 \%$ & $8.6 \%$ & $5 \cdot 4 \%$ & $5 \cdot 0 \%$ & $3 \cdot 2 \%$ & $2 \cdot 7 \%$ & $2 \cdot 1 \%$ & $2 \cdot 6 \%$ & $5.9 \%$ & \\
\hline Feb. & $4.0 \%$ & $62 \cdot 2 \%$ & $2 \cdot 4 \%$ & $5 \cdot 9 \%$ & $7 \cdot 2 \%$ & $4.3 \%$ & $3 \cdot 2 \%$ & $2 \cdot 2 \%$ & $2 \cdot 0 \%$ & $1.8 \%$ & $1.8 \%$ & $2.9 \%$ & \\
\hline Mar. & $1 \cdot 1 \%$ & $4 \cdot 1 \%$ & $76 \cdot 7 \%$ & $3 \cdot 8 \%$ & $3 \cdot 6 \%$ & $2 \cdot 8 \%$ & $1.9 \%$ & $1 \cdot 2 \%$ & $1 \cdot 3 \%$ & $0.7 \%$ & $1 \cdot 2 \%$ & $1.4 \%$ & \\
\hline Apr. & $0 \cdot 4 \%$ & $0 \cdot 4 \%$ & $4 \cdot 3 \%$ & $89 \cdot 0 \%$ & $1.7 \%$ & $1 \cdot 1 \%$ & $0 \cdot 7 \%$ & $0.4 \%$ & $0 \cdot 4 \%$ & $0.4 \%$ & $0.4 \%$ & $0 \cdot 7 \%$ & \\
\hline May & $0 \cdot 3 \%$ & $0 \cdot 4 \%$ & $1 \cdot 3 \%$ & $8.5 \%$ & $85.9 \%$ & $1.2 \%$ & $0.7 \%$ & $0 \cdot 4 \%$ & $0 \cdot 4 \%$ & $0 \cdot 2 \%$ & $0 \cdot 3 \%$ & $0 \cdot 4 \%$ & \\
\hline June & $0 \cdot 3 \%$ & $0 \cdot 5 \%$ & $1.3 \%$ & $2 \cdot 7 \%$ & $8 \cdot 1 \%$ & $83.9 \%$ & $0.7 \%$ & $0 \cdot 5 \%$ & $0 \cdot 4 \%$ & $0 \cdot 4 \%$ & $0 \cdot 4 \%$ & $0.8 \%$ & \\
\hline July & $0 \cdot 4 \%$ & $0 \cdot 5 \%$ & $1.9 \%$ & $3 \cdot 4 \%$ & $5 \cdot 0 \%$ & $9 \cdot 2 \%$ & $\overline{77 \cdot 5 \%}$ & $0.5 \%$ & $0.4 \%$ & $0 \cdot 3 \%$ & $0 \cdot 3 \%$ & $0.6 \%$ & \\
\hline Aug. & $0 \cdot 4 \%$ & $0 \cdot 8 \%$ & $2.4 \%$ & $5 \cdot 5 \%$ & $7 \cdot 4 \%$ & $6 \cdot 2 \%$ & $8.4 \%$ & $65.9 \%$ & $0 \cdot 8 \%$ & $0 \cdot 5 \%$ & $0.6 \%$ & $1.0 \%$ & \\
\hline Sep. & $0.4 \%$ & $0.7 \%$ & $2.9 \%$ & $5.9 \%$ & $8.8 \%$ & $6.4 \%$ & $4.9 \%$ & $6.5 \%$ & $61.4 \%$ & $0.4 \%$ & $0.6 \%$ & $1.2 \%$ & \\
\hline Oct. & $0 \cdot 3 \%$ & $0 \cdot 3 \%$ & $2.9 \%$ & $8 \cdot 1 \%$ & $10 \cdot 4 \%$ & $7 \cdot 5 \%$ & $6 \cdot 0 \%$ & $4.3 \%$ & $6 \cdot 3 \%$ & $52 \cdot 5 \%$ & $0 \cdot 4 \%$ & $0.9 \%$ & \\
\hline Nov. & $0 \cdot 2 \%$ & $0 \cdot 1 \%$ & $1.8 \%$ & $4.6 \%$ & $9 \cdot 1 \%$ & $5 \cdot 8 \%$ & $5 \cdot 1 \%$ & $4.0 \%$ & $3 \cdot 5 \%$ & $4.7 \%$ & $60 \cdot 5 \%$ & $0.4 \%$ & \\
\hline Dec. & $0 \cdot 1 \%$ & $0 \cdot 4 \%$ & $1 \cdot 1 \%$ & $2.9 \%$ & $5.4 \%$ & $4 \cdot 4 \%$ & $2 \cdot 8 \%$ & $2 \cdot 5 \%$ & $2 \cdot 3 \%$ & $2 \cdot 1 \%$ & $5 \cdot 0 \%$ & $70 \cdot 9 \%$ & \\
\hline
\end{tabular}

Fig. 3. Birth month and onset month distribution of hand-foot-mouth disease in Fuyang from 2008 to 2013.

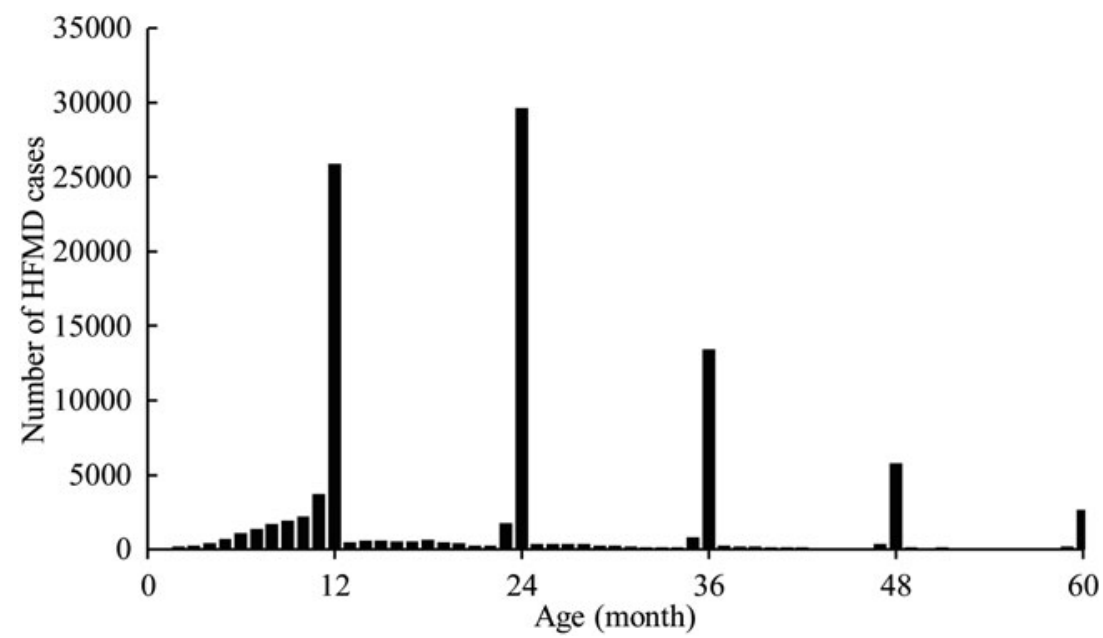

Fig. 4. Age distribution of hand-foot-mouth disease (HFMD) in Fuyang from 2008 to 2013.

with a small sample of reported cases having virus isolated. Clinical samples were collected by medical facilities to confirm the virus serotype based on established procedures [17]. Out of the reported cases, $1032(1 \%)$ were laboratory confirmed, $529(0 \cdot 5 \%)$ were severe, and $34(0 \cdot 03 \%)$ were fatal (22 in 2008 , one in 2009 , five in 2010, none in 2011, one in 2012, and five in 2013). Of laboratory-confirmed cases, EV71, Cox A16 and other enteroviruses accounted for $60 \cdot 1 \%$, $7 \cdot 1 \%$ and $32 \cdot 8 \%$, respectively. 
Table 2. Absolute and relative risk of HFMD infection by birth month in the first 60 months of life with January as the reference group for each birth-month group

\begin{tabular}{lllllll}
\hline \hline Birth month & No. of cases & No. of births & $\begin{array}{l}\text { Absolute } \\
\text { risk }\end{array}$ & RR & $95 \%$ CI & $P$ value \\
\hline January & 434 & 12305 & $3 \cdot 5 \%$ & 1 & - & - \\
February & 448 & 10401 & $4 \cdot 3 \%$ & $1 \cdot 22$ & $1 \cdot 07-1 \cdot 39$ & $0 \cdot 0025$ \\
March & 887 & 9797 & $9 \cdot 1 \%$ & $2 \cdot 57$ & $2 \cdot 30-2 \cdot 87$ & $<0 \cdot 0001$ \\
April & 2352 & 8154 & $28 \cdot 9 \%$ & $8 \cdot 18$ & $7 \cdot 41-9 \cdot 02$ & $<0 \cdot 0001$ \\
May & 2777 & 8042 & $34 \cdot 5 \%$ & $9 \cdot 79$ & $8 \cdot 88-10 \cdot 79$ & $<0 \cdot 0001$ \\
June & 2493 & 8614 & $28 \cdot 9 \%$ & $8 \cdot 21$ & $7 \cdot 44-9 \cdot 05$ & $<0 \cdot 0001$ \\
July & 1665 & 9273 & $18 \cdot 0 \%$ & $5 \cdot 09$ & $4 \cdot 60-5 \cdot 64$ & $<0 \cdot 0001$ \\
August & 947 & 9382 & $10 \cdot 1 \%$ & $2 \cdot 86$ & $2 \cdot 56-3 \cdot 20$ & $<0 \cdot 0001$ \\
September & 610 & 9238 & $6 \cdot 6 \%$ & $1 \cdot 87$ & $1 \cdot 66-2 \cdot 11$ & $<0 \cdot 0001$ \\
October & 522 & 12329 & $4 \cdot 2 \%$ & $1 \cdot 20$ & $1 \cdot 06-1 \cdot 36$ & $0 \cdot 0041$ \\
November & 587 & 13768 & $4 \cdot 3 \%$ & $1 \cdot 21$ & $1 \cdot 07-1 \cdot 37$ & $0 \cdot 0023$ \\
December & 684 & 12156 & $5 \cdot 6 \%$ & $1 \cdot 60$ & $1 \cdot 42-1 \cdot 79$ & $<0 \cdot 0001$ \\
\hline \hline
\end{tabular}

RR, Relative risk; CI, confidence interval.

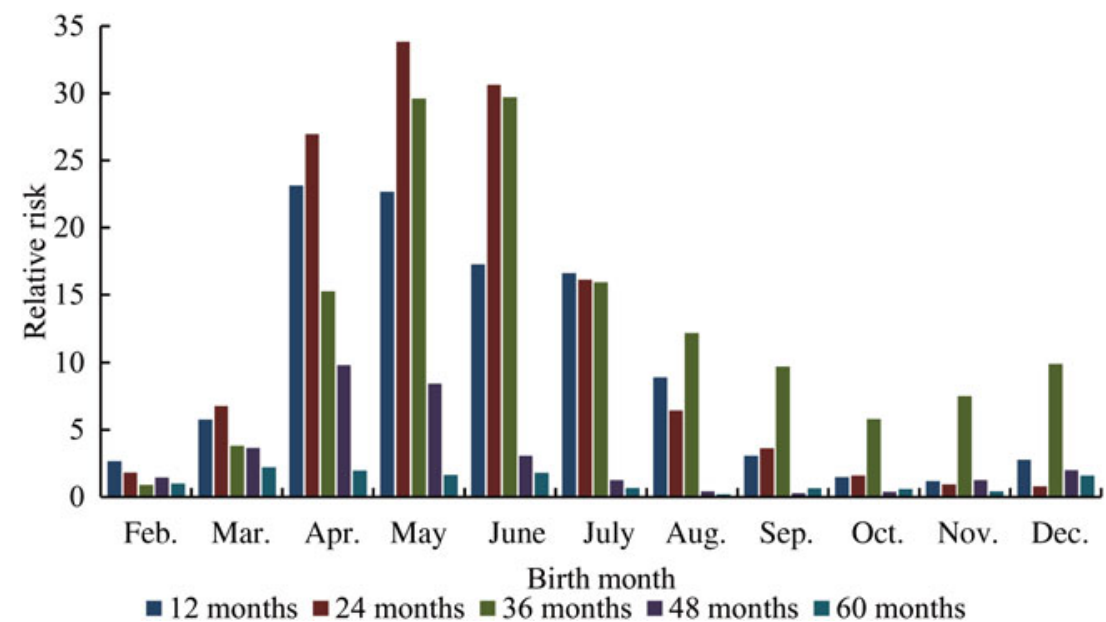

Fig. 5. Relative risk of hand-foot-mouth disease infection by age and birth month with January as the reference month for each age and birth-month group.

EV71-associated cases predominated the laboratoryconfirmed virus serotype for almost every year since 2008. The proportion of patients infected by other enteroviruses demonstrated an upward trend and exceeded EV71 infections in 2012. In contrast, the number of Cox A16 cases were minimal and relatively small. Cox A16 and other enteroviruses were almost equally distributed in 2009 (Fig. 6a).

EV71 infections were more severe than Cox A16 and other enterovirus infections across different age groups, especially in those aged 12, 24, 36, 48 and 60 months. The percentage of other enterovirus infections accounted for $43 \cdot 1 \%$ in other age groups (Fig. 6b).
Figure $6(c, d)$ illustrates that the isolated virus serotype had similar distribution in birth-month and onset-month groups. EV71 predominance also occurred in patients who were born or infected with HFMD between April and June. The majority of September-born/infected patients was due to other enteroviruses.

\section{Spatial distribution}

HFMD affected all 172 regions in Fuyang, but the influence varied by region and time. Figure 7 shows the morbidity map of the HFMD epidemic in Fuyang over the 6-year study period. As shown in Figure 7 , the incidence rate of HFMD increased 

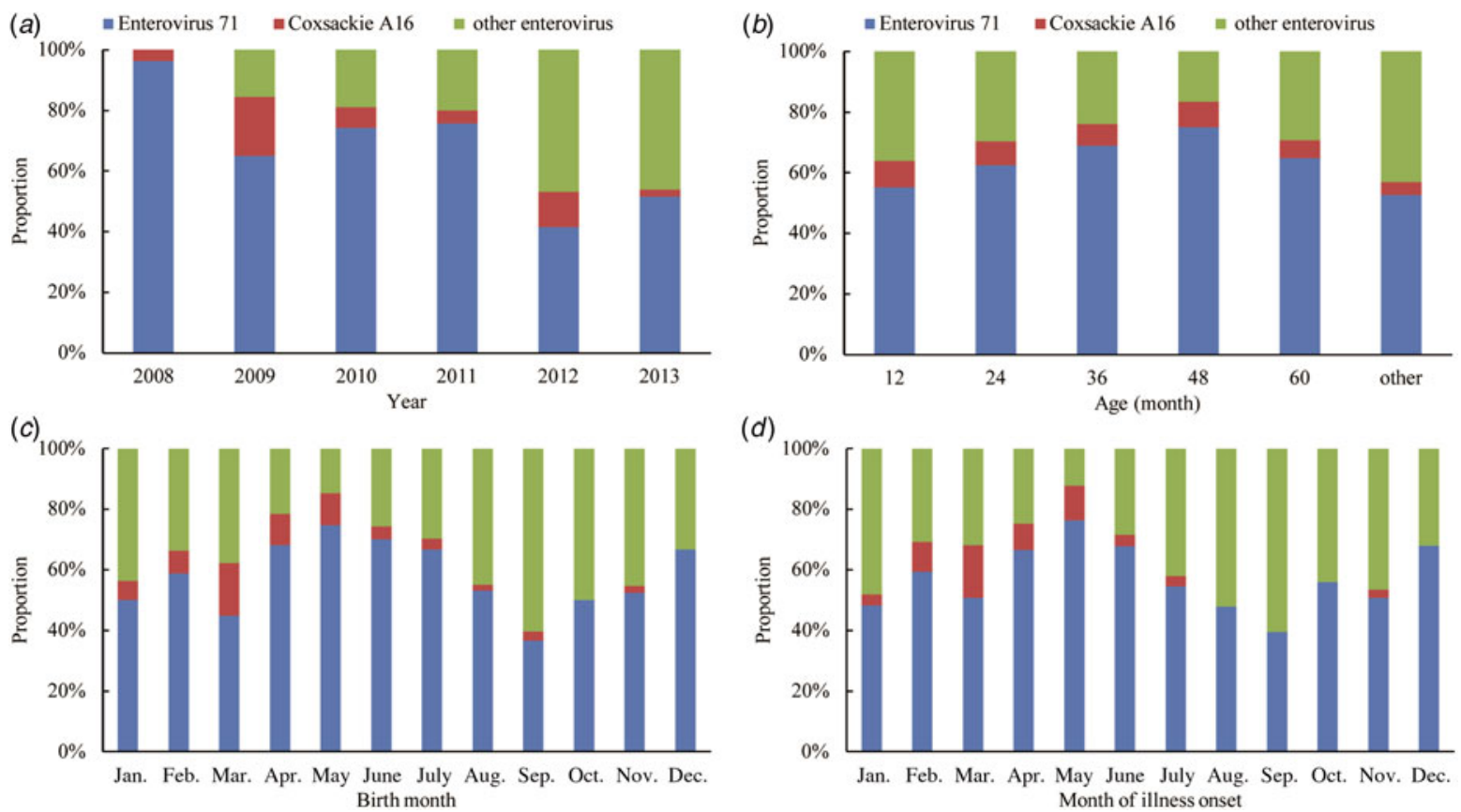

Fig. 6. Virus serotype distribution of hand-foot-mouth disease in Fuyang from 2008 to 2013. (a) Virus serotype distribution by year. (b) Virus serotype distribution by patients' age (month). (c) Virus serotype distribution by patients' birth month. (d) Virus serotype distribution by month of illness onset.
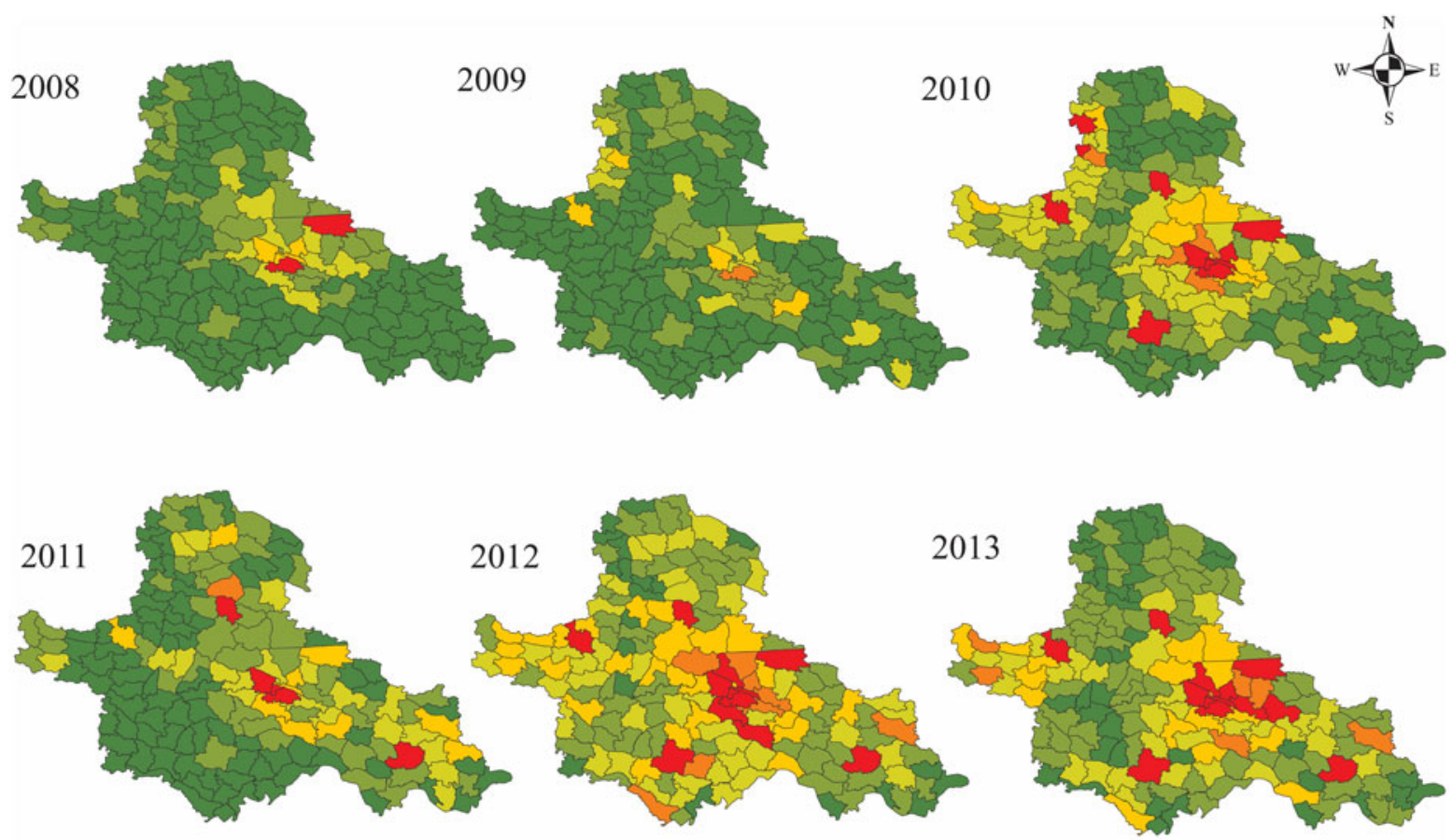

HFMD incidence rate (per 100000 )

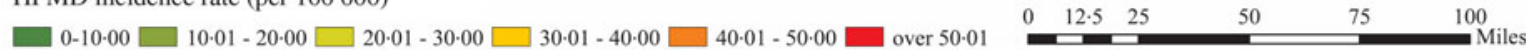

Fig. 7. Morbidity map of hand-foot-mouth disease (HFMD) in Fuyang at the town level from 2008 to 2013. 
Table 3. Most likely clusters of HFMD cases detected using space-time permutation model, 2008-2013

\begin{tabular}{|c|c|c|c|c|c|c|c|}
\hline Year & Cluster centre/radius (km) & Cluster time & $\begin{array}{l}\text { Observed } \\
\text { cases }\end{array}$ & $\begin{array}{l}\text { Expected } \\
\text { cases }\end{array}$ & LLR & $\mathrm{RR}$ & $P$ value \\
\hline 2008 & $\left(32 \cdot 881200^{\circ} \mathrm{N}, 115 \cdot 855998^{\circ} \mathrm{E}\right) / 10 \cdot 47$ & 25 Apr. 2008 to 1 May 2008 & 711 & $494 \cdot 95$ & $44 \cdot 34$ & $1 \cdot 44$ & $<0 \cdot 001$ \\
\hline 2009 & $\left(32.521496^{\circ} \mathrm{N}, 116 \cdot 346684^{\circ} \mathrm{E}\right) / 18.07$ & 28 May 2009 to 2 June 2009 & 78 & $25 \cdot 08$ & $35 \cdot 73$ & $3 \cdot 11$ & $<0 \cdot 001$ \\
\hline 2010 & $\left(33 \cdot 016740^{\circ} \mathrm{N}, 115 \cdot 216394^{\circ} \mathrm{E}\right) / 13 \cdot 12$ & 25 June 2010 to 28 June 2010 & 150 & $69 \cdot 49$ & $35 \cdot 07$ & $2 \cdot 16$ & $<0 \cdot 001$ \\
\hline 2011 & $\left(32 \cdot 605225^{\circ} \mathrm{N}, 116 \cdot 489229^{\circ} \mathrm{E}\right) / 19 \cdot 41$ & 6 Oct. 2011 to 12 Oct. 2011 & 55 & $12 \cdot 82$ & $37 \cdot 97$ & $4 \cdot 29$ & $<0 \cdot 001$ \\
\hline 2012 & $\left(33 \cdot 112986^{\circ} \mathrm{N}, 115 \cdot 297970^{\circ} \mathrm{E}\right) / 11 \cdot 34$ & 1 Apr. 2012 to 7 Apr. 2012 & 191 & $92 \cdot 68$ & $39 \cdot 97$ & $2 \cdot 06$ & $<0 \cdot 001$ \\
\hline 2013 & $\left(33 \cdot 019227^{\circ} \mathrm{N}, 115 \cdot 112160^{\circ} \mathrm{E}\right) / 11.98$ & 26 Mar. 2013 to 1 Apr.2013 & 180 & 68 & $63 \cdot 48$ & $2 \cdot 65$ & $<0.001$ \\
\hline
\end{tabular}

LLR, Log likelihood ratio; RR, relative risk.
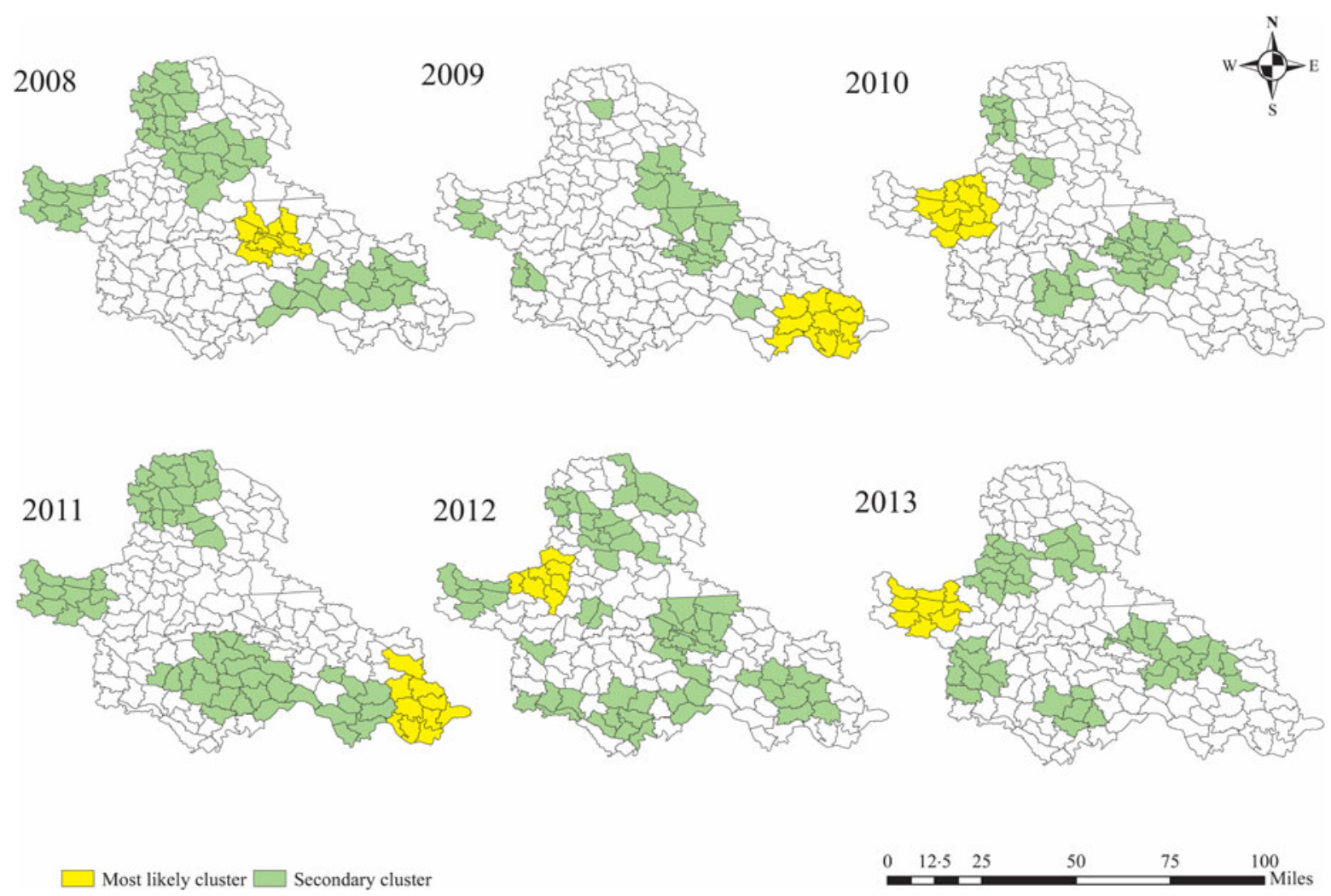

Fig. 8. Cluster map of hand-foot-mouth disease in Fuyang at the town level from 2008 to 2013.

significantly, and the infection continued to spread wider except in 2011. Most epidemic hotspots (incidence rate $>50 \cdot 01 / 10000$ ) were located in urban area, especially in Yingzhou and Yingdong districts. In total, the incidence rate in urban area was higher than that in rural areas over the study period $(31.42$ and $13 \cdot 78 / 10000$, respectively).

\section{Spatio-temporal cluster analysis}

SaTScan $9 \cdot 4$ was utilized to identify the risk regions and time periods of HFMD in Fuyang from 2008 to 2013, and ArcGIS 10.2 was applied to create
HFMD cluster maps (Table 3, Fig. 8). One most likely cluster was detected each year, which was least likely to have occurred by accident [8]. The most likely cluster included 1365 cases over the study period, and was located in different places in Fuyang. In 2009 and 2011, the centre of the most likely cluster was located in almost the same place (Yingshang), but the cluster time was different (between May and June in 2009, October in 2011). The cluster centre resided in Linquan and the surrounding area in 2010, 2012 and 2013, with a similar cluster time in 2012 and 2013 (March-April). The scanning result in 2008 was different from other years, Yingzhou was detected to 
be the most likely cluster at the end of April. The cluster radius stabilized between 10 and $14 \mathrm{~km}$ except for 2009 and 2011 (18.07 and 19.41 km, respectively). The time-frame of the most likely cluster emerged in different months over the entire study period. The largest LLR and RR appeared in 2013 and 2011, respectively. Overall, the scale and RR of the clusters were large in 2009 and 2011, and cluster intensity was strong in 2008 and 2013. Further investigations revealed that the dominant virus serotype in the most likely cluster was EV71 from 2008 to $2013(100 \%, 81 \cdot 3 \%, 83 \cdot 9 \%$, $83 \cdot 1 \%, 48 \cdot 5 \%, 82 \cdot 0 \%$, respectively. Due to the limited number of laboratory-confirmed cases, the proportion was calculated for detected cluster areas within a whole year instead of the cluster time). Furthermore, the proportion of children attending kindergarten within the most likely cluster was $28 \cdot 2 \%$ in 2008 , but was small for the remaining 5 years $(<4 \cdot 0 \%)$.

Several secondary clusters were identified each year in Fuyang, which are less likely to be true clusters [8]. As shown in Figure 8, the number of secondary clusters varied by year, covering different areas with different cluster times. Relative risk for these secondary clusters ranged from 1.44 to 23.08 . The largest and least number of secondary clusters occurred in 2012 and 2010. Nearly half of secondary clusters occurred during April-June (47·4\%).

\section{DISCUSSION}

HFMD is one of the most frequently occurring infectious diseases in China, and half a million to one million patients suffer from this epidemic each year [18]. HFMD characteristics vary with region, time, and spatial scale level. Previous studies have investigated the demographic, spatial, and temporal distributions of HFMD at large or medium spatial-scale levels [9, 11-13]. In China, the county- or city-level CDC is responsible for the implementation of preventive and control measures. Information on HFMD characteristics at the country, province, city, or countylevel is insufficient. In particular, the features of HFMD in Fuyang at the town level remains vague. Since the large HFMD epidemic in China 'originated' from Fuyang city of Anhui Province in 2008, it is worthwhile describing the epidemiology of HFMD in this city. In this study, the distribution of HFMD in Fuyang was examined with respect to time, age in months, birth month, onset month, virus serotype, area, etc. Town level was employed as the basic research unit for spatial and spatio-temporal analyses.
Descriptive analysis revealed a rising incidence rate of HFMD over the study period. April, May and June were the high-epidemic months of HFMD in Fuyang. Possible explanations for such seasonal pattern may be associated with meteorological parameters. HFMD is caused by various human enteroviruses whose survival and propagation are facilitated by a thermal and/or humid environment [2]. Previous studies revealed a significant association between HFMD and specific weather conditions [2, 17-20]. The number of HFMD cases was related to climate factors, such as average temperature and relative humidity, in Singapore, mainland China, Japan, and Hong Kong [2, 17, 19, 20]. Fuyang has a warm, temperate, semi-humid monsoon climate. According to statistical data in recent decades provided by Fuyang Meteorological Bureau, the average temperature increases from $8.8^{\circ} \mathrm{C}$ in March to $27.7^{\circ} \mathrm{C}$ in July and then begins to decline. Therefore, it is likely that weather condition is the most likely reason for the seasonal pattern of HFMD in Fuyang.

The gender ratio in patients with HFMD in Fuyang was 1.8:1. A similar male predominance has been observed in other studies of HFMD from Asia, the gender ratios were approximately $1 \cdot 7: 1$ in Shandong, 1.8:1 in Guangdong, $1 \cdot 5: 1$ in Taiwan and $1 \cdot 3: 1$ in Singapore [11, 12, 21, 22]. Preschool children living at home predominated the reported cases, which is consistent with the findings in Sichuan, Guangdong Province, and Beijing [9, 12, 23]. One possible reason is that children usually start kindergarten at the age of 36 months in China. Most of the children aged $<36$ months are living at home. In the present study, $93.5 \%$ of preschool children living at home were aged $<36$ months. A particular pattern of birth-month and onset-month distribution among HFMD patients aged $<60$ months was observed in the present study. Patients born between April and June were relatively numerous compared to other birth-month groups. Patients had a high risk of HFMD infection in: (1) the birth month; (2) peak months of HFMD; (3) the month before birth month. Cao et al. reported a similar pattern of risk period of HFMD, 'In addition to summer season, HFMD poses a high risk in birth month for a child and the risk of infection decreases as the gap from the birth month increases'. This study was based on 18445 reported HFMD cases in Beijing in 2008 [24]. More research is needed to verify the generality of these findings.

A periodical age (month) distribution of HFMD was detected in the present study. Across the first 60 months of life, the onset of HFMD in Fuyang was 
age-dependent and exhibited a periodic pattern (a 12-month periodicity), with children aged 12, 24 and 36 months being the most susceptible. A duration of about 1-year periodicity of age distribution of HFMD cases was also observed in Beijing [24]. However, the age-specific vulnerable groups (12, 24, 36 months) have not been explicitly identified. Whether the age (month) distribution pattern of HFMD is universal remains uncertain.

We could use the above-mentioned findings regarding birth-month and onset-month distribution to explain the observed age distribution in Figure 4. The risk of developing HFMD in birth month is very high, which will inevitably lead to high frequencies in children aged 12, 24, 36, 48, and 60 months. For example, if a patient is born in January, 12, 24 or 36 months later, it is still January (i.e. the birth month of the patient). Conversely, from Figure 4, it could be found that a child would be more susceptible to HFMD in the first, second and third birth month (i.e. 12, 24 and 36 months). Recent study has established that high maternal antibody levels in serum and in breast milk confer protection against enterovirus infection, and enterovirus antibody levels in breast milk wane over time [25]. In the present study, the HFMD frequencies increase with age in patients under aged $<11$ months (cf. Fig 4), which could be explained by the same principle.

Across the first 60 months of life, children born between April and June had an eight- to nine-fold higher risk of HFMD infection than children born in January; the risk, relative to an individual's birth month and age, was highest for 24-month-olds born in May (RR 34·85, 95\% CI 26.49- 43.25). In the literature, the age- and birth-month disease associations have been found in respiratory syncytial virus infections and other diseases [26, 27]. However, to the best of our knowledge, research reporting the impact of birth month and age on the risk of HFMD infection is very limited for pertinent cross-referencing.

Unlike in Guangdong Province, Singapore, and Beijing where the predominating enterovirus changes with time [12, 22, 23], EV71 has been the dominant virus serotype (compared to Cox A16) in Fuyang since 2008. An entirely opposite pattern has been observed in Hong Kong, with Cox A16 always making up the majority of the reported cases [28]. The main virus serotype in those aged $12,24,36,48$, or 60 months as well as in patients who were born or infected between April and June was EV71. In the case of other enteroviruses, it was the predominant strain in 2012 and was prevalent in September-born/ infected patients.

In the present study, the HFMD incidence rate in urban area was higher than that in rural areas in Fuyang, which is consistent with findings of previous studies [13, 24]. However, more HFMD cases were reported in rural areas. A possible reason for this finding is the high population density in urban areas. Over the study period in Fuyang, the average density of population was 357 inhabitants $/ \mathrm{km}^{2}$ in urban areas and 204 inhabitants $/ \mathrm{km}^{2}$ in rural areas. Moreover, urban and rural areas have different diagnostic capacity, timeliness and accuracy of reporting, which make the urban surveillance system more effective and might lead to the higher incidence rate in urban areas [13].

After the descriptive analysis, SaTScan was utilized to perform a spatio-temporal cluster analysis of HFMD in Fuyang. ArcGIS 10.2 was used to visualize the results. Town-level scan results indicated that the time-frame, scale, RR, and intensity of the most likely cluster varied over the study period. The most likely cluster was located in Yingzhou in 2008, Yingshang in 2009 and 2011, Linquan and its surrounding area (Jieshou) in 2010, 2012 and 2013. Generally, the most likely cluster was formed during the peak months of HFMD (April-June) except for 2011, so the time period of detected clusters is reasonable. EV71 dominated the virus serotype in most clusters, which is consistent with the findings in Shandong Province [11]. The change of proportion of children attending kindergarten in a most likely cluster may be associated with relevant effective public health action. The largest number of secondary clusters occurred in 2012, which may be related to the highest HFMD incidence rate in this year (cf. Figs 1 and 8). Preventive interventions towards detected spatiotemporal hotspots might help to reduce HFMD incidence. Moreover, knowledge on the characteristics of these clusters could be useful to identify high-risk clusters in the future. However, it should be noted that the spatial scan statistics are based on the assumption of circular or cylinder scanning windows to detect potential clusters, which may not represent the actual shape of the clusters [23]. We plan to explore finer methods to conduct spatio-temporal analysis.

There are several limitations in this study. First, potential reporting bias might exist in the surveillance data. HFMD cases could be underreported because of the self-limiting nature of this disease, or patients 
who did not seek medical care. Second, further analyses stratified by virus serotype are constrained by the small proportion of laboratory-confirmed cases (only $1 \%$ of reported cases were laboratory confirmed in the surveillance data). Third, the population assumption made to calculate the disaggregated incidence rates and the hypothesis of no HFMD-related population migration. The demographic data at town level were unavailable, which may reduce the accuracy of spatial distribution analysis of HFMD at town level. This was not necessarily a problem for other analyses in the study. The relative risks for each age and birth-month group were estimated by monthly birth population in 2008 and surveillance data of HFMD. Missing migration information could affect estimated risks to some extent. Last, due to the limited access to surveillance data in wide areas, some conclusions arising from this study such as the upward trend incidence, the spatial distribution and spatio-temporal clusters of HFMD, might be region-specific and thus restrict the large-scale application.

\section{CONCLUSIONS AND SUGGESTIONS}

To summarize, the present study offers information that is essential in understanding the epidemiological characteristics and spatio-temporal patterns of HFMD in Fuyang over the period 2008-2013. This research provides important bases for the prediction of pandemic trends, such as high-risk season, population, and areas. Key findings in the study are as follows: (1) the peak months of HFMD are April-June; (2) the onset of HFMD is age-dependent and exhibits a 12-month periodicity, with children aged 12, 24 and 36 months being the most vulnerable groups; (3) birth month and age might be risk factors of HFMD infection, with children born between April and June being 8-9 times more at risk compared to the January birth-month group, and 24-month-olds born in May had the highest relative risk compared to the reference January birth-month group; (4) a large proportion of laboratory-confirmed cases were associated with EV71.

To our knowledge, only one study by Cao et al. [24] refers to the possible birth-month disease and age disease association in HFMD researches. In their study, although these authors noted a periodical age distribution in HFMD patients, the specific age groups were not determined precisely [24]. Therefore, further studies are warranted to confirm whether the detected pattern and associations are universal. More in-depth insights from medical and biological perspectives are needed to elucidate the inherent mechanism underlying the relationship between birth month/age and HFMD in future studies.

In view of current prevention and control measures and findings in the present study, we would suggest that: (1) enhancement of laboratory infrastructures to expand the capacity of serotype identification. More clinically reported HFMD cases are needed to be virus isolated for further data analysis; (2) preschool children living at home require more public attention and medical resources; (3) for children aged $<60$ months, special attentions should be paid to age-specific groups (12, 24, 36 months) and birthmonth groups (children born between April and June). Clinicians and families could be made aware of the increased likelihood of HFMD in birth month, peak months of HFMD and perhaps the month before birth month; (4) in addition, as suggested by Sadeharju et al., exclusive breastfeeding is also important in reducing the risk of enterovirus infections in infants [25].

Currently, multiple groups and medical scientists in Singapore, Taiwan and mainland China remain committed to developing safe and effective EV71 vaccines [29-31]. Innovative progress and critical barriers to implementation co-exist in researches. The first EV71 vaccine was approved by the China Food and Drug Administration on 3 December 2015 [31]. However, EV71 vaccination cannot prevent infections caused by Cox A16 and other enteroviruses [32], which limits the wide application of EV71 HFMD vaccine. A surveillance system is also needed to assess the long-term impact of the introduction and deployment of the vaccine. Results in current study may provide new perspectives for developing multivalent vaccines against HFMD by taking into account the disease incidence pattern with respect to birth month and age. In addition, the foregoing findings will help regulatory authorities to formulate and implement efficient vaccination strategies. For example, targeted vaccination towards high-risk birth-month and age groups may contribute to herd immunity.

\section{ACKNOWLEDGEMENTS}

The authors are grateful to the Editor and four reviewers for providing very helpful suggestions and comments. We thank Fuyang Centre for Disease Control and Prevention for providing HFMD surveillance data from 2008 to 2013. Gratitude is also 
extended to Fuyang Civil Affairs Bureau for providing the administrative map. This research was supported by the National Natural Science Foundation of China (grant no. 71571 176) and the Research Grants Council of Hong Kong (grant no. T32-102/ $14 \mathrm{~N})$. All authors contributed equally to this work.

\section{DECLARATION OF INTEREST}

None.

\section{REFERENCES}

1. World Health Organization. A Guide to Clinical Management and Public Health Response for Hand, Foot and Mouth Disease (HFMD). Geneva: WHO Press, 2011.

2. Hii YL, Rocklov J, Ng N. Short term effects of weather on hand, foot and mouth disease. PLoS ONE 2011; 6: e16796.

3. Solomon T, et al. Virology, epidemiology, pathogenesis, and control of enterovirus 71. Lancet Infectious Diseases 2010; 10: 778-790.

4. Chan L, et al. Deaths of children during an outbreak of hand, foot, and mouth disease in Sarawak, Malaysia: clinical and pathological characteristics of the disease. Clinical Infectious Diseases 2000; 31: 678-683.

5. Chen KT, et al. Epidemiologic features of hand-footmouth disease and herpangina caused by enterovirus 71 in Taiwan, 1998-2005. Pediatrics 2007; 120: e244 e252.

6. Chen $\mathbf{J}$, et al. Geovisual analytics to enhance spatial scan statistic interpretation: an analysis of US cervical cancer mortality. International Journal of Health Geographics 2008; 7: 57.

7. Kulldorff M, et al. Evaluating cluster alarms: a spacetime scan statistic and brain cancer in Los Alamos, New Mexico. American Journal of Public Health 1998; 88: $1377-1380$.

8. Kulldorff M. SaTScan ${ }^{\mathrm{TM}}$ user guide for version 9.4, 2015 (http://www.satscan.org/). Accessed 8 March 2015.

9. Liu L, et al. Spatio-temporal clustering of hand, foot and mouth disease at the county level in Sichuan province, China, 2008-2013. Epidemiology and Infection 2015; 143: 831-838.

10. Kulldorff M, et al. A space-time permutation scan statistic for disease outbreak detection. PLoS Medicine 2005; 2: 216.

11. Liu Y, et al. Detecting spatial-temporal clusters of HFMD from 2007 to 2011 in Shandong Province, China. PLoS ONE 2013; 8: e63447.

12. Deng T, et al. Spatial-temporal clusters and risk factors of hand, foot, and mouth disease at the district level in Guangdong Province, China. PLoS ONE 2012; 8: e56943-e56943.

13. Qi Z, et al. Surveillance of hand, foot, and mouth disease in mainland China (2008-2009). Biomedical and Environmental Sciences 2011; 24: 349-356.
14. Haining RP. Spatial Data Analysis: Theory and Practice. Cambridge: Cambridge University Press, 2003, pp. 258.

15. YIN F. A study on the applicability of space-time scan statistic in early-warning of infectious disease (Dissertation). Chengdu, Sichuan, China: Sichuan University, 2007, 13 pp.

16. Tango T, Takahashi K. A flexibly shaped spatial scan statistic for detecting clusters. International Journal of Health Geographics 2005; 4: 11.

17. Xing W, et al. Hand, foot, and mouth disease in China, 2008-12: an epidemiological study. Lancet Infectious Diseases 2014; 14: 308-318.

18. Wang J, et al. Hand, foot and mouth disease: spatiotemporal transmission and climate. International Journal of Health Geographics 2011; 10: 1-10.

19. Onozuka D, Hashizume M. The influence of temperature and humidity on the incidence of hand, foot, and mouth disease in Japan. Science of the Total Environment 2011; 410: 119-125.

20. Ma E, et al. Is hand, foot and mouth disease associated with meteorological parameters? Epidemiology and Infection 2010; 138: 1779-1788.

21. Chen SC, et al. An eight-year study of epidemiologic features of enterovirus 71 infection in Taiwan. The American Journal of Tropical Medicine and Hygiene 2007; 77: 188-191.

22. Ang LW, et al. Epidemiology and control of hand, foot and mouth disease in Singapore. Annals of the Academy of Medicine, Singapore 2009; 38: 106-112.

23. Wang $\mathbf{J}$, et al. Epidemiological analysis, detection, and comparison of space-time patterns of Beijing hand-footmouth disease (2008-2012). PLoS ONE 2014; 9: e92745.

24. Cao Z, et al. An epidemiological analysis of the Beijing 2008 hand-foot-mouth epidemic. Chinese Science Bulletin 2010; 55: 1142-1149.

25. Sadeharju K, et al. Maternal antibodies in breast milk protect the child from enterovirus infections. Pediatrics 2007; 119: 941-946.

26. Lloyd PC, et al. The effect of birth month on the risk of respiratory syncytial virus hospitalization in the first year of life in the United States. Pediatric Infectious Disease Journal 2014; 33: e135-e140.

27. Boland MR, et al. Birth month affects lifetime disease risk: a phenome-wide method. Journal of the American Medical Informatics Association 2015; 22: 10425.

28. Ma E, et al. Changing epidemiology of hand, foot, and mouth disease in Hong Kong, 2001-2009. Japanese Journal of Infectious Diseases 2010; 63: 422-426.

29. Hwa SH, et al. Preclinical evaluation of the immunogenicity and safety of an inactivated enterovirus 71 candidate vaccine. PLoS Neglected Tropical Diseases 2013; 7: e2538.

30. Cheng A, et al. A Phase I, randomized, open-label study to evaluate the safety and immunogenicity of an enterovirus 71 vaccine. Vaccine 2013; 31: 2471-2476.

31. Mao QY, et al. EV71 vaccine, a new tool to control outbreaks of hand, foot and mouth disease (HFMD). Expert Review of Vaccines 2016 (in press).

32. Liu CC, et al. Prospect and challenges for the development of multivalent vaccines against hand, foot and mouth diseases. Vaccine 2014; 32: 6177-6182. 